\title{
Preservação e valorização do património natural e científico de Angola: águas termais da montipa, Namibe
}

\section{Preservation and valorisation of the natural and scientific heritage of Angola: the thermal springs of montipa, Namibe}

\author{
DOI: $10.46932 / \mathrm{sfjdv2n2-197}$
}

Received in: March 1st, 2021

Accepted in: May 30th, 2021

\author{
Agostinho Francisco Cachapa \\ Doutorado em Ciências Químicas pela Universidade de la Laguna, Reino de Espanha. \\ Professor Catedrático da Universidade do Namibe. \\ Current Institution: Universidade do Namibe - Angola. \\ Address: Universidade do Namibe, Farol da Noronha, Moçâmedes, Namibe, Angola. \\ E-mail: agostinhocachapa@yahoo.es
}

\section{Teresa de Jesus Portelinha Almeida Patatas}

Pós-doutoranda em Educação Comparada. Doutorada em Ciências da Educação pela Universidade Lusófona, Lisboa-Portugal.

Professora Auxiliar da Universidade do Namibe. Investigadora Associada do Centro de Estudos Interdisciplinar de Educação e Desenvolvimento (CeiED).

Current Institution: Universidade do Namibe - Angola.

Address: Universidade do Namibe, Farol da Noronha, Moçâmedes, Namibe, Angola.

E-mail: teresapatatas.angola@gmail.com

\section{RESUMO}

Angola é um país com inúmeros patrimónios naturais e científicos, com destaque para o património termal. No município da Bibala, província do Namibe, existe a necessidade de preservação e valorização deste tipo de património, que compreende áreas de importância preservacionista, biodiversidade singular de valor universal e beleza cénica, constituindo habitat natural de excepcional importância científica, turística e terapêutica, contudo pouco divulgado e investigado. Assim, a presente comunicação tem como objectivo dar a conhecer as águas termais da Montipa, e, a necessidade de valorização e a preservação deste admirável património. Durante os anos 2015 e 2016 realizou-se uma investigação para a caracterização deste recurso hidrotermal, do ponto de vista geoquímico e ambiental. Os resultados mostraram que estas águas termais podem ser aplicadas em diversas áreas, com distintas finalidades, ainda pouco exploradas. Também revela a necessidade de reforçar a divulgação das já reconhecidas qualidades terapêuticas locais. Este recurso está a ser subaproveitado e negligenciado. Nesta base, torna-se imperioso preservar este património; promover os seus benefícios para o aumento da qualidade de vida local; divulgar as suas características científicas e possíveis finalidades; e, incentivar a investigação académica nesta área do saber. Tendo em conta o alvo nacional de diversificação da economia, este recurso hidrotermal pode ser visto como um meio de obtenção de receitas, angariação de investimentos (nacionais e internacionais) e de desenvolvimento provincial. Outrossim, é imprescindível sensibilizar as entidades competentes para a necessidade de medidas efectivas para a concretização destes desideratos.

Palavras-chaves: Património natural angolano, Património científico, Águas termais da Montipa, Namibe. 


\begin{abstract}
Angola is a country with innumerable natural and scientific assets, with emphasis on the thermal heritage. In the Bibala municipality, a province of Namibe, there is the need of preservation and valorisation of this type of heritage, which entails areas of preservation importance, singular biodiversity of universal value, and scenic beauty, constituting a natural habitat of exceptional scientific, touristic and therapeutical importance, however little divulged and researched. Thus, the present communication aims to raise awareness about the thermal springs of Montipa, and the need to value and preserve this admirable heritage. During 2015 and 2016, research on the characterisation of this hydrothermal resource was carried out, from a geochemical and environmental point of view. The results had shown that these thermal waters can be applied in diverse areas, with distinct purposes, still underexplored. They also expose the need to strengthen the dissemination of the already known local therapeutical qualities. This resource is being underexploited and neglected. Having this context in consideration, it becomes imperative to preserve this heritage; to promote its benefits in order to improve the local quality of life; to divulge its scientific characteristics and possible purposes; and to encourage academic research in this area. Taking into account the national goal of economic diversification, this hydrothermal resource can be seen as a way of raising revenue, gathering investments (national and international), and developing the province. Likewise, it is crucial to sensitise the competent authorities to the need of effective measures in order to achieve these goals.
\end{abstract}

Keywords: Angolan natural heritage, Scientific heritage, Thermal springs of Montipa, Namibe.

\title{
1 NOTA INTRODUTÓRIA
}

A divulgação qualquer tipo de património para a sociedade, é um passo muito marcante no sentido da sua preservação, pois quanto mais se saber sobre o bem patrimonial, maior número de pessoas e de entidades se envolverá na sua defesa, na sua valorização. Deste modo, este património se tornará parte da identidade dos sujeitos de uma determinada área.

O património possui uma condição de legado que integra os bens de uma colectividade, definidos pelo seu valor científico, estético, educativo, recreativo, terapêutico, cultural e simbólico, sem esquecer todos aqueles voltados para o bem comum. O património inclui componentes de carácter diverso, utilizados pelas comunidades humanas para fins socioeconómicos, este é também o caso do património termal da Montipa.

O município da Bibala, na localidade da Montipa, possui um recurso hidrotermal que, valorizado como atractivo turístico pode ser mais utilizado para práticas relacionadas com o lazer, a recreação e a terapia. Este património termal pode criar um ponto de práticas turístico-recreativas, fazendo com que a Montipa seja reconhecida como um destino desejável. Pode oferecer ao turismo uma actividade socioeconómica, proporcionando uma maior divulgação do património natural e local. Assim, o presente trabalho tem como objectivo dar a conhecer as águas termais da Montipa, e, a necessidade de valorização e a preservação deste bem patrimonial. 
A comunicação está dividida em quatro partes, sendo a primeira uma contextualização sobre o património natural, científico e termal, águas termais, termalismo e desenvolvimento turístico local; na segunda parte encontram-se os aspectos metodológicos para obtenção dos dados da comunicação; na terceira parte a exposição e discussão dos resultados e na última parte apresentam-se as conclusões.

\section{REFERENCIAL TEÓRICO: ÁGUA VERSUS PATRIMÓNIO NATURAL, CiENTÍFICO E CULTURAL}

O património deve ser deliberado com base no que dizia Ruiz (1996), como constituído por elementos materiais e imateriais, naturais ou culturais, herdados do passado ou criados no presente, no qual um determinado grupo de indivíduos reconhece sinais de sua identidade. Pode-se dizer que o património, segundo Álvarez-Vasquez e Uña-Álvarez (2015, p. 1), “integra todos os bens de uma colectividade, definidos pelo seu valor científico, estético, educativo, recreativo, simbólico e cultural”.

Como parte do património, segundo a Organização das Nações Unidas para a Educação, Ciência e Cultura ( UNESCO) (UNESCO, 2005), o natural se define como as formações geológicas e fisiológicas, zonas de interesse naturais e as zonas estritamente delimitadas que constituem habitat de espécies animais e vegetais ameaçadas, com valor universal excepcional do ponto de vista da ciência ou da conservação ou beleza natural. O valor cultural do património define-se como grupos de construções isoladas ou reunidos que, em virtude da sua arquitetura, unidade ou integração na paisagem que têm valor universal excepcional do ponto de vista da história, da arte ou da ciência (Gambarota, Leonardi e Elias, 2018), ou como diz a UNESCO (s.d.), são obras do homem ou obras conjuntas do homem e a natureza, assim como as zonas, incluídos os lugares arqueológicos, que tenham um valor universal desde o ponto vista histórico, estético, etnológico ou antropológico.

Património científico, também chamado de património cultural de ciência e tecnologia (C \& T), se considera "o conhecimento científico e tecnológico produzido pelo homem, além de todos aqueles objectos (inclusivé documentos em suporte papel), colecções arqueológicas, etnográficas e espécimes das coleções biológicas que são testemunhos dos processos científicos e do desenvolvimento tecnológico.” (Granato, 2009, p. 79). Ainda se pode acrescentar neste conceito, aqueles lugares ou zonas os quais podem ser utilizados para estudos de carácter científicos e académicos.

Neste contexto, as águas são consideradas como um património natural, por fazerem parte de territórios e espaços e também servir de base económica e política de determinados grupos sociais, ainda são fontes de identificação cultural e referência simbólica. (Catullo \& Brodin, 2015). E, porque estes têm uma história, um passado (Quintela, 1999) e servem como objecto de investigação cientifica. Neste diapasão, é muito importante ter em conta com o que, as Nações Unidas, no relatório sobre os recursos 
hídricos (2006), afirma que as águas são patrimónios que se podem converter no bem mais caro da história da humanidade e ser mais motivo de guerras do que o ouro negro: o petróleo.

\section{3 ÁGUAS TERMAIS COMO PATRIMÓNIO TERMAL E TURÍSTICO}

As águas termais são águas subterrâneas mineralizadas e aquecidas provenientes de rochas. As águas termais são bastante importantes, visto que possuem características que lhes conferem propriedades de reabilitação, tratamento e promoção da saúde. Por este facto, elas são muito procuradas para proporcionar o bem-estar às populações e criam o valor turístico deste património. Assim sendo surge o turismo termal que consiste em viagens para uso e desfrute dos patrimónios naturais da água minero medicinal e termal e seus derivados por orientação médica em instalações para o seu uso. Por isso, a necessidade da sua valorização e preservação como algo singular do património balnear, fundamentalmente na melhoria da saúde humana, baseada no termalismo.

O termalismo está relacionado com o património águas termais, na sua utilização para fins terapêuticos e de todo o seu entrono, desde os gases que emanam da fonte até às lamas. Assim, se pode definir Termalismo, segundo Grácio (2016, p. 41), “como um conjunto de práticas onde a água termal tem a função de agente terapéutico".

\section{METODOLOGIA}

Desde o ponto de vista metodológico, realizou-se uma pesquisa bibliográfica e documental sobre os aspectos relacionados com águas termais e termalismo, património natural, termal, científico e cultural, sem esquecer as questões das potencialidades turísticas, relacionando-os com este caso em particular da área da Montipa. Depois se fez várias visitas de campo ao local para observação, onde se tirou algumas fotos e para recolha de amostras de águas, em várias épocas durante os anos de 2015 e 2016, no tempo quente e no tempo do frio, para análise tanto in situ $(\mathrm{pH}$, condutividade, turvação e temperatura de emergência) como ao nível laboratorial.

Trata-se de um estudo de caso, da zona da Montipa, visto que como afirma Meirinhos e Osório (2010, p. 16), "é frequentemente referido como permitindo estudar o objecto (caso) no seu contexto real, utilizando múltiplas fontes de evidência (qualitativas e quantitativas) e enquadra-se numa lógica de construção de conhecimento, incorporando a subjectividade do investigador”.

\subsection{COLHEITAS, ACONDICIONAMENTO E TRANSPORTE DE AMOSTRAS.}

A colheita de amostra de água é uma das etapas mais importantes no momento da qualidade de um corpo hídrico. A confiabilidade dos resultados e sua interpretação adequada dependem da sua correcta 
execução. No caso dos colectores superficiais, geralmente são utilizados frascos de vidro de borossilicato (V e VB) ou de polietileno (PE). Para o nosso trabalho, utilizaram-se frascos de vidro e de PE, que foram previamente esterilizados, como recomendados por Tiago (2017).

\subsection{ANÁLISES DOS PARÂMETROS FÍSICOS E QUÍMICOS}

As determinações de parâmetros organoléticos, físicos e químicos da água, como a temperatura, condutividade eléctrica (CE), turvação, cor, cheiro e sabor devem ser, preferencialmente realizados em campo (in situ), imediatamente após a colheita, para prevenir alterações nas suas propriedades, usando como recurso os medidores multiparametros portáteis de alta precisão, hoje existentes no mercado. Os sólidos totais dissolvidos (STD) e os parâmetros químicos ( $\mathrm{pH}$, ferro, alumínio, magnésio e manganés, dureza total, amónio, cloro residual livre, nitrato, cloretos, sulfatos) foram analisados no laboratório de água na cidade do Lubango, segundo os procedimentos da Organização Mundial de Saúde (OMS). (OMS, 2010).

\section{CARACTERIZAÇÃO DA LOCALIDADE DA MONTIPA}

A localidade da Montipa encontra-se localizada a Norte do município da Bibala próximo à margem do Rio Peça (Puilangombe). A área é constituída por 11 bairros e terá sido descoberta por um caçador chamado Tchimôngua, originário dos povos Muimbas na área do Iona.

Esta área, corresponde à da divisão topográfica de Angola limitada, pelos paralelos 14`30` S e

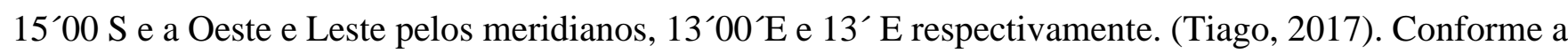
Figuras 1 e 2:

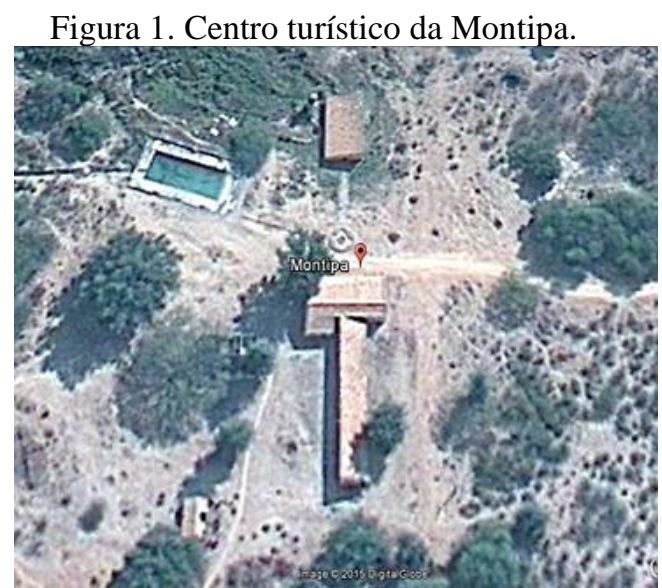

Fonte: Google earth.

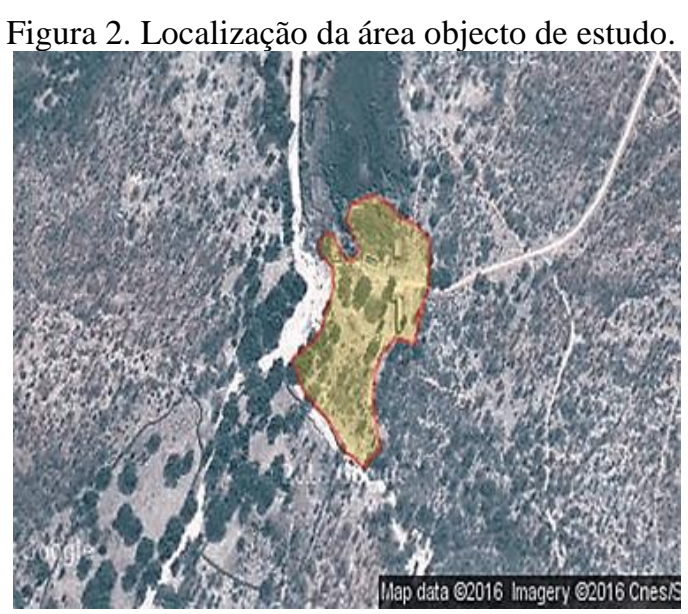

Fonte: Google earth

A capela Soful, na povoação da Montipa, foi construída em 1955, por uma organização portuguesa e passaram ao controlo do Governo Angolano depois de 1975. (Tiago, 2017). Conforme a Figura 3: 
Figura 3. Representa a fundação da área de estudo.

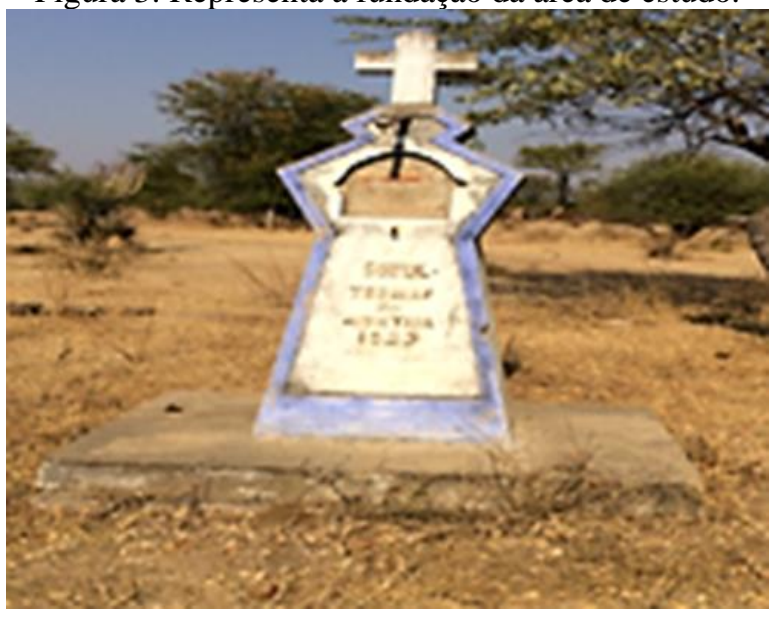

Fonte: Tiago, 2017.

\section{GEOLOGIA E HIDROGEOLOGIA}

A área da Montipa está directamente relacionada com a enorme fenda NW-SE que coincide com o rio Puilangombe e encontra-se sobre uma fenda menor de descompressão com direcção NE-SW que intercepta a primeira fenda. (Pereira, Van-Dunem \& Tassinari, 2006).

A Missão de Pedologia de Angola (1963, citado por Fernandes, 2014) distinguiu, de entre outras, a zona geológica que se estende de Oeste para Leste, com rochas ígneas pós-pérmicas, na qual se destacam os doloritos olivínicos, e não olivínicos. A zona essencialmente está formada por rochas eruptivas ou sedimentares altamente metamorfizadas, com especial realce para as graníticas.

Desde o ponto de vista hidrogeológico, a mineralização das águas aumenta, por faixas paralelas de $\mathrm{E}$ ao $\mathrm{W}$, bruscamente nos interflúvios igualmente aos vales aluvionares dos cursos de água que envolvem a Escarpa da Chela e que engloba as zonas de Cainde, Bibala e Lola. (Victor, J.; Pinto, C.; Almeida, L.; Calejo, M. \& Veríssimo, L., 2007).

\section{CLIMATOLOGIA}

A área da Montipa, como parte do município da Bibala, as precipitações anuais atingem os 800900 mm, devido à subida forçada das correntes atmosféricas provocada pela cadeia montanhosa. A estação chuvosa tem a duração de cinco meses (Novembro a Março). (Victor et al., 2007).

O município da Bibala tem a temperatura média anual, rondando os $23,7^{\circ} \mathrm{C}$. Cfomo demonstardo na Figura 4. 


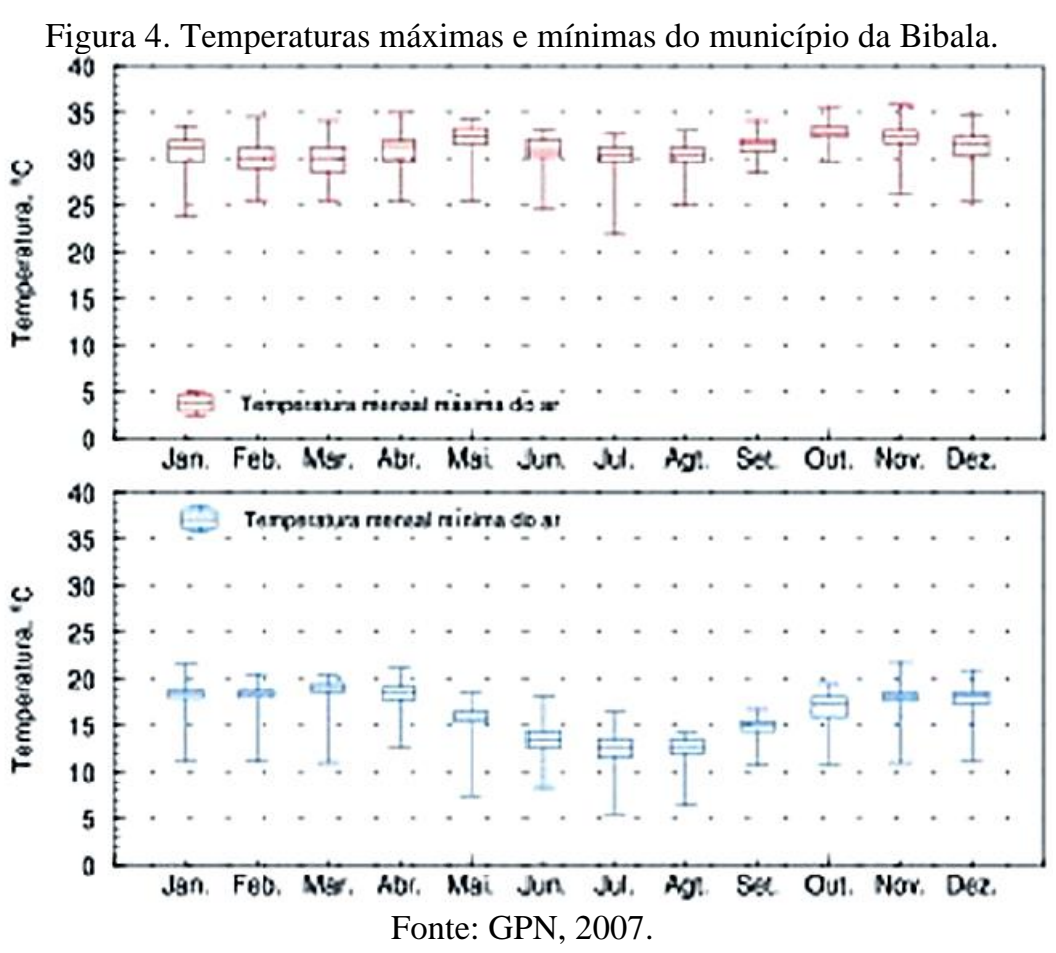

A humidade relativa anual no município é de 58,1\%, ocorrendo os valores máximos em Março e os mínimos em Novembro e Dezembro. De acordo com a classificação climática de Thorntwaite, o clima da Bibala é semi-húmido seco $(\mathrm{C} 1)$, marcando a transição para os planaltos do interior, com clima húmido (Victor et al., 2007).

\section{RESULTADOS E DISCUSSÃO}

Para a apresentação dos resultados e discussão mostram-se primeiro os parâmetros físicos e depois os parâmetros químicos deste recurso hidrotermal da Montipa.

\section{PARÂMETROS FÍSICOS}

Trata-se de uma água límpida, sem cheiro e sem sabor, que emerge a uma temperatura média entre $48-49^{0} \mathrm{C}$ e se desconhece a sua extensão (Tiago, 2017).

Este património termal foi descoberto em 1955, por uma organização aquando da construção da capela do Soful (Tiago, 2017).

A conductividade é uma propriedade relacionada com o grau de mineralização da água, e é de 625 $\mu$ S/cm. (Tiago, 2017), e é de mineralização média, segundo Hindi (1999).

A turvação é considerada como um factor importante para estimar a qualidade da água relacionada com o seu grau de transparência e limpeza, logo a água da Montipa é considerada clara e transparente, segundo a classificação de Massol (2010). 
As águas termais da Montipa consideram-se hipertermal, já que emergem com uma temperatura superior a $37^{\circ} \mathrm{C}\left(48^{\circ} \mathrm{C}\right)$, pelo facto, está água pode ser aplicada para fins terapêuticos, pela acção térmica sobre o organismo humano. (Torres, 2006, p. 52). E segundo Armijo e Martin, (1994), pode ser utilizada concretamente como sedante e combate de contracções e hipertonia muscular. No local, há uma outra piscina, em que a temperatura é mais baixa, esta pode ser utilizada para outros fins, tais, como:

- Estimulante;

- Melhoria da circulação de órgãos internos;

- Activação das mudanças metabólicas;

- Acção dermatológica;

- Exerce a acção vasoconstrictora.

As águas termais da Montipa, por apresentar valores de STD entre 300 e $450 \mathrm{mg} / \mathrm{L}$ e com base em a quantidade de STD, classificam-se, segundo o Livro Branco (2010, citado por Santos, 2011, p. 51), em águas hipomineralizadas. (STD no rango de 500 e 1500 mg/L), e segundo Davis e Dewiest (1966), são consideradas como água doce e levemente salina.

\section{PARÂMETROS QUÍMICOS}

Estes resultados dos parâmetros químicos analisados se espelham na Tabela 1 abaixo:

Tabela 1. Resultados das análises dos parâmetros químicos.

\begin{tabular}{|c|c|c|}
\hline PARÂMETRO & VALOR & UNIDADE \\
\hline pH & 8.2 & Escala de Sorenson \\
\hline $\mathbf{F e}$ & 0.2 & $\mathrm{mg} / \mathrm{L} \mathrm{Fe}$ \\
\hline Al & 0,01 & $\mathrm{mg} / \mathrm{L} \mathrm{Al}$ \\
\hline Mn & 0.0 & $\mathrm{mg} / \mathrm{L} \mathrm{Mn}$ \\
\hline $\mathrm{NO}_{3}^{-}$ & 6.5 & $\mathrm{mg} / \mathrm{L} \mathrm{NO}_{3}^{-}$ \\
\hline $\mathrm{NH}_{4}{ }^{+}$ & 0.01 & $\mathrm{mg} / \mathrm{L} \mathrm{NH}_{4}^{+}$ \\
\hline $\mathrm{SO}_{4}^{2-}$ & $>400$ & $\mathrm{mg} / \mathrm{L} \mathrm{SO}_{4}{ }^{2-}$ \\
\hline Dureza total & 80 & $\mathrm{mg} / \mathrm{L} \mathrm{CaCO}_{3}$ \\
\hline Cloro residual & 0.0 & $\mathrm{mg} / \mathrm{L} \mathrm{Cl}_{2}$ \\
\hline
\end{tabular}

Fonte: Autores.

Com base nos resultados, que se apresentam na Tabela 1 , pode-se aferir que a água termal que emerge na zona da Montipa, é mineral e natural. Apresenta as seguintes características:

$\checkmark$ Trata-se de uma água alcalinas por ter carácter básico, com um pH maior que 7; 
$\checkmark \mathrm{O} \mathrm{pH}$, para além de controlar a maior parte das reacções químicas na natureza, controla, também, e a actividade biológica é, na maior parte dos casos, apenas possível para valores de $\mathrm{pH}$ compreendidos em 6 e 8 .

$\checkmark$ Embora muitas substâncias possam contribuir para a alcalinidade de uma água, a maior parte desta característica nas águas naturais é devida aos hidróxidos $(\mathrm{OH})$, carbonatos $\left(\mathrm{CO}_{3}{ }^{2-}\right)$ e bicarbonatos $\left(\mathrm{HCO}_{3}{ }^{-}\right)$.

De acordo com os resultados obtidos, as características alumínicas desta água são quase nulas devido a sua reduzida concentração de alumínio.

Em relação à concentração do ferro, existe uma certa quantidade que se pode ter em conta, que segundo Rojas, Fortich, e Pavajeau, (2014), esta água pode ser aplicada ao corpo humana, por ingestão para reforçar a hemoglobina.

A dureza (em geral expressa em $\mathrm{mg} / \mathrm{L}$ de carbonato de cálcio $\mathrm{CaCO}_{3}$ ) de uma água é devida à presença de catiões metálicos bivalentes principalmente cálcio $\left(\mathrm{Ca}^{2+}\right)$ e magnésio $\left(\mathrm{Mg}^{2+}\right)$. Estes iões metálicos bivalentes estão, em geral, associados com o anião bicarbonato $\left(\mathrm{HCO}_{3}{ }^{-}\right)$, sulfato $\left(\mathrm{SO}_{4}{ }^{2-}\right)$, cloreto $\left(\mathrm{Cl}^{-}\right)$e nitrato $\left(\mathrm{NO}_{3}^{-}\right)$(Sousa, 2001).

De acordo com os resultados obtidos, a água térmica da Montipa, classifica-se como água moderadamente dura, com base na classificação de Massol (2010). Esta característica faz com que esta água tenha solubilidade dos compostos metálicos.

Em relação ao anião sulfatos, este resultado mostra altas concentrações de minerais sulfatados na fonte, podendo esta água ser aplicada como purgante, quando ingerida. Segundo Rojas, Fortich, e Pavajeau (2014) pode ser utilizada para a melhoria da saúde humana, ao nível gastrintestinal.

\section{APROVEITAMENTO DESTE PATRIMÓNIO TERMAL}

A água térmica da Montipa trata-se de um património com elevado potencial turístico, porque para além da água que emerge quente, há uma envolvente natural com determinadas características potenciais para o ecoturismo.

Existe uma grande importância em conhecer as propriedades deste recurso hidrotermal, já que o estudo determina o aproveitamento ou o benefício que esta água pode proporcionar ao município em particular e a província em geral. Os resultados encontrados mostram um imenso potencial para o seu aproveitamento em actividades de turismo de saúde, através de banhos na água quente, proporcionando acções terapêuticas na pele (balneoterapia) e interior do organismo (ingestão). 
Assim, com base na sua composição físico e química, água térmica da Montipa, podem ser indicados para tratar enfermidades do foro reumático, dermatológico e estados de esgotamento psicofísico.

Apesar do grande potencial deste património, a sua valorização e preservação são quase que nulas, independentemente, de ser aproveitado por alguns visitantes.

\section{A VALORIZAÇÃO E PRESERVAÇÃO DO PATRIMÓNIO TERMAL DA MONTIPA}

Tendo em conta, o estado e aproveitamento que se dá, e pode se dar, às águas termais da Montipa e do seu entorno, é pertinente e imperioso valorizar e preservar este património termal. Para esse alvo deve-se envolver a comunidade, visto que o adequado aproveitamento deste recurso contribui para o desenvolvimento humano e melhoria da qualidade de vida.

Para isso, será necessário, começar com a implementação na comunidade de um processo de educação patrimonial, que é, segundo Surya (2009, p. 6), "processo permanente e sistemático de trabalho educacional centrado no património como fonte primária de conhecimento e enriquecimento individual e colectivo". Isso se pode realizar, com base no seguinte:

- Promoção de uma relação de afectiva da comunidade pelo património, despertando assim um processo de aproximação da população ao património, à memória, de forma agradável, prazerosa e lúdica;

- Levar as crianças e adultos a um processo activo de conhecimento, apropriação e valorização de sua herança natural e termal;

- Capacitar a todos para um melhor usufruto desses bens que as Termas da Montipa oferecem.

Há necessidade de realização de iniciativas, por parte da Administração Municipal, do Governo da Província e das comunidades, no sentido da valorização e preservação deste património hidrotermal, nomeadamente:

- Elaboração de uma brochura ou uma cartilha para a divulgação do património;

- Inclusão destas termas nos pacotes turísticos provincial e/ou nacional;

- Melhoria do acesso rodoviário Bibala-Montipa;

- Implementação de zonas de protecção, no sentido de prevenir, reduzir e controlar a poluição da água termal deste local;

- Implementação de projectos e actividades contínuas com estudantes de diversos níveis de ensino do município.

- Realização de eventos de carácter científico, cultural e turístico para se abordar a importância desta água para o desenvolvimento local; 
O local, objecto de estudo, é de gestão privada, que se encontra em estado de "semiabandono", é sem dúvida, um património que necessita de intervenção urgente, no sentido da sua preservação e valorização. Neste sentido, este espaço poderia beneficiar de um projecto para reabilitação total na vertente terapêutica (balneários) e no aproveitamento dos espaços naturais (turismo ecológico), que circundam a fonte termal, para dar um valor acrescentado para o desenvolvimento sustentável, qualitativo, socioeconómico, das comunidades e do município da Bibala.

\section{CONSIDERAÇÕES FINAIS}

O presente trabalho, apesar de ser uma investigação preliminar, pretende contribuir para o estudo do património natural e termal da Montipa e a sua valorização. Neste diapasão, reconhece a importância do turismo, enquanto estratégia da preservação e valorização do património e desenvolvimento local.

Assim, a caracterização do local da fonte termal, e dela como tal, mostra as qualidades deste património natural que pode contribuir para o desenvolvimento sustentável e turístico das comunidades, do município da Bibala, da Província do Namibe, sendo por isso, urgente a sua preservação e valorização.

A preservação e a valorização deste património natural e termal é importante para um melhor conhecimento do mesmo e permitiria ao mesmo tempo restituir a importância que este sítio merece, com base na relação histórica que teve e que tem com a comunidade circundante, possibilitando ao mesmo tempo colocar o mesmo num sentido mais global ao nível da importância do termalismo para a saúde humana no nosso país.

Num período, em que no país se evidencia a diversificação da economia, é de bom tom, que promova o turismo. Acredita-se que o trabalho realizado é uma mais valia, no sentido de trazer uma visão de como alavancar o desenvolvimento provincial e melhorar a qualidade vida da população da área da Montipa.

O estudo pode e poderá servir de base e referência a futuras investigações relacionadas com a temática do patrimonio natural e termal e novas propostas de itinerários e roteiros que incluam a incomparável Montipa. 


\section{REFERÊNCIAS BIBLIOGRÁFICAS}

Álvarez-Vázquez, M. \& Uña-Álvarez, E. (2015). Integración del patrimonio natural en el área termal de Ourense (Galicia): importancia de las formas erosivas fluviales. Congreso Internacional del Agua Termalismo y Calidad de Vida. Campus da Auga, Ourense, España.

Armijo \& Martin, S. (1994). Aguas bicarbonatadas. in: Curas Balnearias y Climáticas. Talasoterapia y Helioterapia. Madrid: Ed. Complutense, 261-267.

Catullo, M. \& Brodin, D. (2015). Las aguas termales como patrimonio tangible y soporte de prácticas. Ciudad Nueva Federación (Argentina) y municipio de Santo Amaro da Imperatriz (Brasil). Astrolabio. Número 5. Córdoba: Universidad Nacional de Córdoba. ISSN: 16687515.

Davis, S. N \& Dewiest, R. J. M. (1996). Hydrogeology. New York: Wiley.

Fernandes, J. (2014). As pinturas do abrigo do Tchitundu-Hulu Mucai. Um contributo para o conhecimento da arte rupestre da região. Vol I. Tese de mestrado. Tomar: Instituto Politécnico de Tomar - Universidade de Trás-os-Montes e Alto Douro.

Gambarota, D., Leonardi, V. \& Elías, S. (2018). Las aguas termales como patrimonio natural y cultural. Potencialidad turística en Bahía Blanca (Argentina). International Journal of Scientific Management and Tourism. 4-2, 285-301.

Grácio, A. (2016). Turismo Termal em São Pedro do Sul. Dissertação de Mestrado em Lazer, Património e Desenvolvimento da Faculdade de Letras da Universidade de Coimbra. Coimbra: Universidade de Coimbra.

Granato, M.; Maia, E. \& Santos, F. (Jul./Dez. 2014). Valorização do patrimônio científico e tecnológico brasileiro: descobrindo conjuntos de objetos de C\&T pelo Brasil. Anais do Museu Paulista: História e Cultura Material, 22 (2), 11-34. Acessado 04 de Junho de 2018. Disponível em https://dx.doi.org/10.1590/S0101-47142014000200002.

Hindi, E. C. (1999). Caracterização Hidroquímica e Hidrogeológica das fontes cársticas das bacias dos rios Tumiri, água comprida, fervida e das onças - Colombo, PR. Dissertação (Mestrado) Curitiba: Universidade Federal do Paraná, Curitiba.

Massol, A. (2010). Curso microbiología sanitaria. Mayagüez: Universidad de Puerto Rico.

Medeiros, M. \& Surya, L. (2009). A Importância da educação patrimonial para a preservação do patrimônio. Fortaleza: ANPUH - XXV Simpósio Nacional DE História.

Meirinho, M. \& Osório, A. (2010). O estudo de caso como estratégia de investigação em educação. EDUSER: Revista de Educação, Inovação, Investigação em Educação. 2(2). Bragança: Instituto Politécnico de Bragança. ISSN 1645-4774.

Naciones Unidas (2006). El agua, una responsabilidad compartida. $2^{\circ}$ Informe de las Naciones Unidas sobre el Desarrollo de los Recursos Hídricos en el Mundo. Programa Mundial de Evaluación de los Recursos Hídricos. Acessado a 03 de Junho de 2018. Disponível em http://unesdoc.unesco.org/images/0014/001444/144409S.pdf. 
Organização Mundial de Saúde (OMS). (2010).

Pereira, E.; Van-Dunem, M. V.; Tassinari, C. C. G. (2006). Notícia explicativa da carta geológica de Bibala. Folha 335. Sul D-33. N-III. Luanda: Instituto Geológico de Angola.

Quintela, M. (2011). Curar e recrear em águas termais: um diálogo etnográfico entre Portugal (Termas de São Pedro do Sul e Termas da Sulfúrea) e Brasil (Caldas da Imperatriz). Anuário Antropológico [Online], II |, Posto online em 24 novembro 2015. Acessado a 04 de Junho de 2018. URL: http://journals.openedition.org/aa/1151; DOI: 10.4000/aa.1151.

Rojas, E., Fortich, M., \& Pavajeau, H. (2014). Determinación del origen y la composición de las aguas termales ubicadas en los municipios de Becerril (Cesar) y Ciénaga (Magdalena), Colombia. Ingenium, 8 (21), 35-42.

Ruiz, J. C. (Septiembre 1996). ¿Hacia una nueva definición de patrimonio histórico? PH Boletín del Instituto Andaluz del Patrimonio Histórico, n. XVI, Sevilla: IAPH.

Santos, (2011). Propriedades e aplicações dermatológicas das águas termais. Porto: Universidade Fernando Pessoa. Acessado a 29 de Janeiro de 2016. Disponível em http://bdigital.ufp.pt/bitstream/10284/2438/3/MONOGRAFIA.pdf.

Sousa, E. (2001). Noções sobre qualidade da água. Lisboa: Instituto Superior Técnico.

Tiago, E. (2017). Caracterização física da água termal da Montipa. Trabalho de fim de curso de licenciatura em Engenharia Ambiental. Namibe: Escola Superior Politécnica do Namibe

Organização das Nações Unidas para a Educação, Ciência e Cultura (UNESCO). (2005). Carpeta de información sobre el patrimonio mundial. Paris.

Organização das Nações Unidas para a Educação, Ciência e Cultura (UNESCO). (s.d.). Indicadores UNESCO de cultura para el desarrollo: Patrimonio. Manual metodológico. Acessado a 04 de Junho de 2018. Disponível em http://es.unesco.org/creativity/sites/creativity/files/iucd_manual_metodologico_1.pdf

Victor, J.; Pinto, C.; Almeida, L.; Calejo, M. \& Veríssimo, L. (2007). Plano de Desenvolvimento Integrado da Província do Namibe 2007-2013. Estudos Sectoriais. Estrutura Biofísica. Vol. 3. Namibe: Governo Provincial do Namibe.

World Health Organization in collaboration with Food and Agriculture Organization of the United Nations, (2007). Safe preparation, storage and handling of powdered infant formula - Guidelines. 